\title{
Simultaneous measurements of the flow velocities in a microchannel by wide/evanescent field illuminations with particle/single molecules
}

\author{
Hongwei Gai, ${ }^{a}$ Ying Li, ${ }^{a}$ Zhanhua Silber-Li, ${ }^{b}$ Yinfa Ma*ac and Bingcheng Lin*a \\ Received 27th October 2004, Accepted 7th February 2005 \\ First published as an Advance Article on the web 24th February 2005 \\ DOI: 10.1039/b416476h
}

\begin{abstract}
A laser-induced fluorescence imaging method was developed to simultaneously measure flow velocities in the middle and near wall of a channel with particles or single molecules, by selectively switching from the wide field excitation mode to the evanescent wave excitation mode.

Fluorescent microbeads with a diameter of $175 \mathrm{~nm}$ were used to calibrate the system, and the collisions of microbeads with channel walls were directly observed. The $175 \mathrm{~nm}$ microbeads velocities in the main flow and at $275 \mathrm{~nm}$ from the bottom of the channel were measured. The measured velocities of particles or single molecules in two positions in a microchannel were consistent with the calculated value based on Poiseuille flow theory when the diameter of a microbead was considered. The errors caused by Brownian diffusion in our measurement were negligible compared to the flow velocity. Single $\lambda$ DNA molecules were then used as a flowing tracer to measure the velocities. The velocity can be obtained at a distance of $309.0 \pm 82.6 \mathrm{~nm}$ away from bottom surface of the channel. The technique may be potentially useful for studying molecular transportation both in the center and at the bottom of the channel, and interactions between molecules and microchannel surfaces. It is especially important that the technique can be permitted to measure both velocities in the same experiment to eliminate possible experimental inconsistencies.
\end{abstract}

\section{Introduction}

Single molecule detection (SMD) technology has made remarkable progress within this decade. ${ }^{1-8}$ It has been demonstrated to be a unique way to exploit fundamental physical and chemical principles of molecules down to a single molecule level and can lead to significant applications for biological and medical studies in the near future. SMD has been applied for monitoring molecular diffusion in free solutions, ${ }^{9}$ studying absorption and desorption of protein molecules at a solid/liquid interface, ${ }^{10,11}$ finding anomalous radial migration of DNA molecules in capillary electrophoresis. $^{12,13}$ In addition to fundamental investigations, SMD has been extensively applied for biological or biochemical areas, such as high throughput DNA screening in electrophoresis, ${ }^{14,15}$ single molecule immunoassay, ${ }^{16}$ DNA sizing and sorting, ${ }^{17-20}$ DNA mapping ${ }^{21,22}$ and in situ hybridization. ${ }^{23}$ The single molecule detection technique was also used to probe the flow profile. For example, Rigler and co-workers $^{24}$ demonstrated a high-spatial resolution flow profile in microchannels by scanning microchannels with diffraction limited laser focusing. The authors reported a detection of single tetramethylrhodamine labeled biomolecules at various locations within a microchannel and demonstrated parabolic flow in two dimensions (i.e. Poiseuille laminar flow). The determination with the single molecule detection technique was validated and proved that SMD could be an important complementary method for traditional particle

*bclin@dicp.ac.cn (Bingcheng Lin) image velocimetry (PIV). However, the near wall velocity of single molecules was not measured.

PIV is a major and well-established technique for measuring velocity fields in macroscopic and microscopic fluid systems. $^{25,26}$ The positions of flowing particles are recorded at two defined times and then the displacement of the particle is calculated by correlating the particle image pairs. The PIV experiments conducted in a microfluidic chip were demonstrated by Santiago et al. ${ }^{27,28}$ to implement a micro-PIV set up and obtain spatially resolved velocity measurements in pressure flow and electrokinetic flow devices. Kwok et al. ${ }^{29}$ used Shah Convolution Fourier Transform Detection to estimate velocities of a single-component particle.

Both single molecule detection techniques and PIV introduced above, however, were not used to measure the flow velocity closer than $450 \mathrm{~nm}$ from the walls. Very recently, a nano-PIV technique was reported by Yoda et al. by only using evanescent waves to obtain the particle velocity of flow field near-wall and to study the electro-osmotic flow in microchannel. ${ }^{30,31}$ However, there has been no system for assuring the velocities from the center to the bottom of a microchannel were accurately measured.

In order to simultaneously measure velocities in the middle and at bottom of a channel, we developed a laser-induced fluorescence imaging system that can be easily switched between the evanescent wave excitation mode and commonly the wide field excitation mode. The $175 \mathrm{~nm}$ fluorescence microbeads and $\lambda$ DNA molecules were used respectively to visualize flow field and to measure velocities. The effect of Brownian diffusion on the measurement was also discussed. This system allows one to measure the flow velocities in the 
middle and at the bottom of a channel in one experiment, which will be a great help for researchers to observe the molecular transport behavior in the whole channel.

\section{Experimental section}

\section{Materials}

The $175 \mathrm{~nm}$ fluorescence microspheres (or microbeads) $($ Ex./Em. $=505 \mathrm{~nm} / 515 \mathrm{~nm})$ were purchased from Molecular Probes (PS-speck Microscope Point Source Kit, Eugene, OR, USA). Doubly distilled water was used. $\lambda$ DNA samples (48,502 bp, Takara Biotechnology, Dalian, China) were labeled with YOYO-I (Molecular Probes, Eugene, OR, USA) at a ratio of one dye molecule per five base-pairs. The concentration of DNA stock solution was 109 pM. Samples were further diluted to $1.09 \mathrm{pM}, 0.109 \mathrm{pM}$ and $0.0109 \mathrm{pM}$ prior to the start of the experiments.

\section{Microchannel assembly}

A fused-silica microchip, custom manufactured by Alberta Microelectronics Corp. (Alberta, Canada), was used for all experiments. The chip channel was $10 \mu \mathrm{m}$ deep, $300 \mu \mathrm{m}$ wide and $3.5 \mathrm{~cm}$ long (Fig. 1A). The chip was placed on the hypotenuse face of a right-angle fused-silica prism (Melles Griot, Irvine, CA; Prism UVGSFS, $A=B=C=2.54 \mathrm{~cm}$ ). The chip and the prism were index-matched with a drop of type FF immersion oil (R. P. Cargille Laboratories, Inc., Cedar Grove, NJ). The prism and the microchannel were fixed to a sturdy holder to minimize position shift from sample loadings. Before each experiment, the channel was washed successively with $10 \mu \mathrm{L}$ sodium hydroxide $(1.0 \mathrm{M}), 10 \mu \mathrm{L}$ water, $10 \mu \mathrm{L}$ hydrochloric acid $(1.0 \mathrm{M})$ and $10 \mu \mathrm{L}$ water.

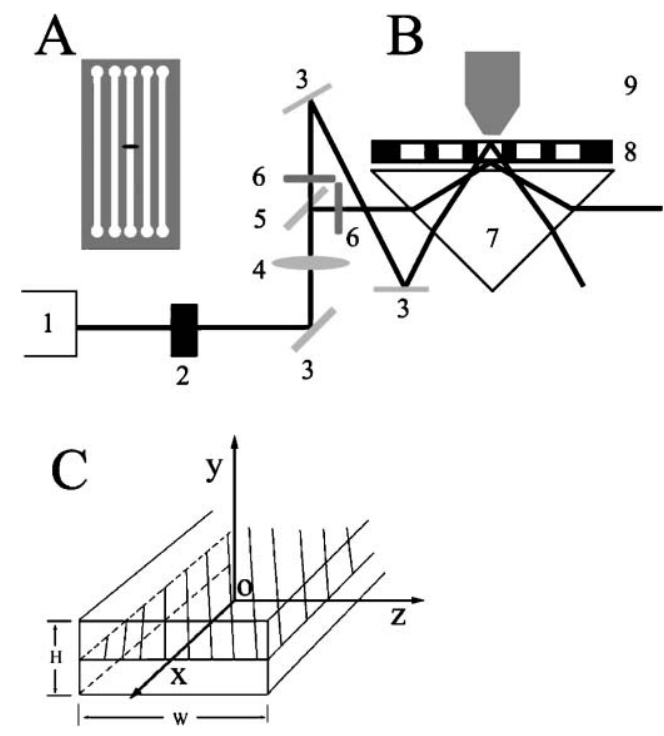

Fig. 1 Optical setup for measuring the velocity of particles and molecules in a microchannel. (A) Top view of the fused silica chip used in the experiments; (B) Sketch of the evanescent wave illumination and wide field lighting. 1, laser; 2 , shutter; 3 , mirrors; 4 , lens; 5 , beamsplitter; 6, gobos; 7, prism; 8, chip; 9, objective; (C) Coordinate of the channel. $X$-axis is the flow direction; $Y$-axis is the channel height; $Z$-axis is the channel wide; $\mathrm{O}$ locates in the plane of $H / 2$ and $W / 2$.

\section{Equipment setup}

The experimental setup for this study is similar to the previous setup with some changes ${ }^{15}$ as shown in Fig. 1B. An electron multiplier CCD (EMCCD, iXon87, Andor Technology, North Ireland) camera was mounted on top of a Zeiss Axioskop upright microscope. The digitization rate of the camera was $5 \mathrm{MHz}$ (12 bits) with the software controller gain set at 255 and the camera was maintained at $-60{ }^{\circ} \mathrm{C}$. The camera was operated in the internal synchronization mode and in the frame-transfer mode for molecular tracking. The excitation source was a water-cooled argon ion laser (488 nm; Spectra Physics, Mountain View, CA, USA). Extraneous light and plasma lines from the laser were eliminated prior to its entry into the observation region with the aid of an equilateral dispersing prism and an optical pinhole. The laser beam passed through a mechanical shutter with programmable control (LS2Z2, Vincent Associates, Rochester, NY, USA) and was focused with a $20 \mathrm{~cm}$ focal length lens (Melles Griot, Irvine, CA, USA). A beamsplitter (Factory Affiliated of Shanghai University of Technology, Shanghai, China) was used to split the laser beam into two orthogonal beams. The horizontal beam was directed to the right angle fused-silica prism $(n=1.46)$ at an angle of about $40^{\circ}-45^{\circ}$ with respect to the normal on to one of the legs of the surfaces of the prism. This beam is refracted through the prism and undergoes total internal reflection (TIR) with an angle of incidence $\theta \approx 74^{\circ}$ at the bottom of the fused silica channel-sample interface. The vertical laser beam reflected through two mirrors, passed through the prism and reflected at the top of the microchannel. This laser beam is out of focus because of its longer propagation distance than the horizontal one. These two laser beams could be selectively obstructed by the gobos (dark plastic plates) placed in the light paths. One $488 \mathrm{~nm}$ holographic notch filter (Kaiser Optical, Ann Arbor, MI; HNFP) with optical density of $>6$ and a $514 \mathrm{~nm}$ bandpass filter (Melles Griot, Irvine, CA) was placed between the objective and the EMCCD. The Zeiss $20 \times / 0.75$ NA plan Apochromat microscope objective and $40 \times / 0.75 \mathrm{NA}$ plan Neofluar microscope objective were used to collect the fluorescence from the fluorescence microbeads and $\lambda$ DNA molecules within evanescent field and wide field, respectively.

An evanescent wave is generated by the total internal reflection (TIR) of light at the interface between two media which have different refractive indices when a parallel beam of light propagates to the interface from the dense medium with refractive index $\left(n_{2}\right)$ to the less dense medium with refractive index $\left(n_{1}\right)$ and the incident angle $\theta$ exceeds the critical angle

$\theta_{\mathrm{c}}=\operatorname{arcsine}\left(n_{2} / n_{1}\right)$. The penetration distance $z_{\mathrm{p}}$ away from the interface located at $z=0$ is calculated using the following formula: ${ }^{32}$

$$
z_{\mathrm{p}}=\frac{\lambda_{0}}{4 \pi \sqrt{n_{2}^{2} \sin ^{2} \theta-n_{1}^{2}}}
$$

Here, $\lambda_{0}$ is the wavelength of the light. In our experiments, the laser at $\lambda_{0}=488 \mathrm{~nm}$ was focused at an angle of incidence of $40^{\circ} \sim 45^{\circ}$ onto the fused silica prism $\left(n_{2}=1.46\right)$. The laser beam was refracted through the prism at an angle of incidence $\theta=71^{\circ} \sim 74^{\circ}$ at the fused silica-water $\left(n_{1}=1.33, \theta_{\mathrm{c}}=66^{\circ}\right)$ 
interface in the microchannel. The penetration distance $z_{\mathrm{p}}$ is $107 \sim 89$ nm. Fig. 2 shows the comparison of $\lambda$ DNA molecules (1.09 pM, pH 2.0) fluorescence imaging with different illumination modes in the microchannel. The whole channel was lit as a bright band at wide field excitation mode and almost all molecules that passed through the view field were excited, as shown in Fig. 2A, in which single molecules could not be distinguished. When the evanescent wave was used to excite the same sample with a $40 \times$ objective, the single $\lambda$ DNA molecules adsorption on to the channel surface were clearly observed in Fig. 2B as reported by Kang et al. ${ }^{33}$ With these results, we verify that the evanescent wave was successfully created in our setup.

\section{Procedures}

The fluorescence microbeads were diluted with deionised water ten fold. The concentration of microbeads was about $3 \times 10^{-8}$ beads $\mathrm{mL}^{-1}$. One microliter microbead solution or $\lambda$ DNA samples were added into one of the reservoirs at the end of the channel and another one was maintained empty. The difference in liquid levels at the two reservoirs was used to drive microbeads or $\lambda$ DNA molecules flowing in the microchannel. When the flow was steady, the images were first recorded with an evanescent wave to acquire the flowing velocity of the near-wall. Then the gobo, which was originally placed in the vertical laser beam (refer to Fig. 1B), was removed so that the wide field excitation mode was used to acquire the bulk velocity.

\section{Results and discussion}

Flow velocities observed by wide/evanescent field with microbeads

When microbeads passed through the microchannel, they were illuminated with evanescent wave mode and then wide field mode. Fig. 3A shows the typical fluorescence images of microbeads flowing in a microchannel driven by hydrostatic pressure when an evanescent wave was used for excitation. Single arrowhead and double arrowhead represent two microbeads in a series of images, respectively. From two consecutive images in which the fluorescence of microbead was captured, the velocity of the microbead was calculated by migration
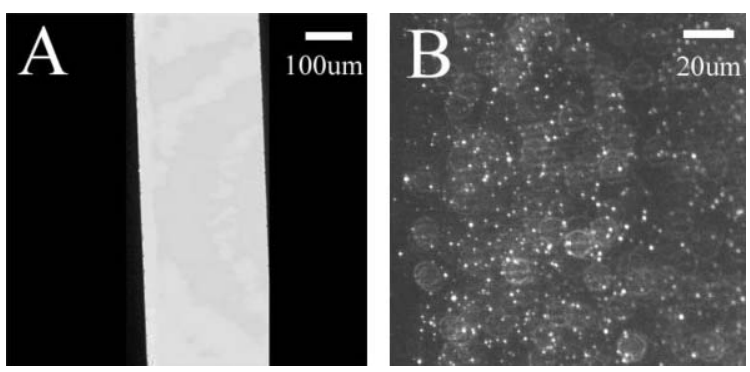

Fig. 2 Chip-based fluorescence imaging of $\lambda$ DNA molecules (1.09 pM, pH 2.0) labeled with YOYO-I under two different excitation modes with $488 \mathrm{~nm}$ laser. (A) Wide field excitation mode with $10 \times$ objective; (B) evanescent wave mode with $40 \times$ objective. (a)
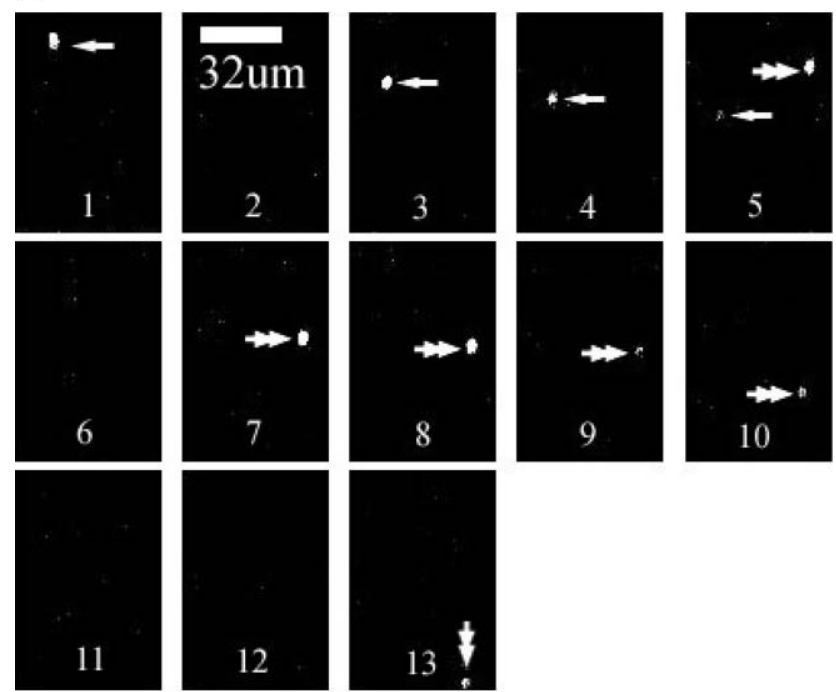

(b)
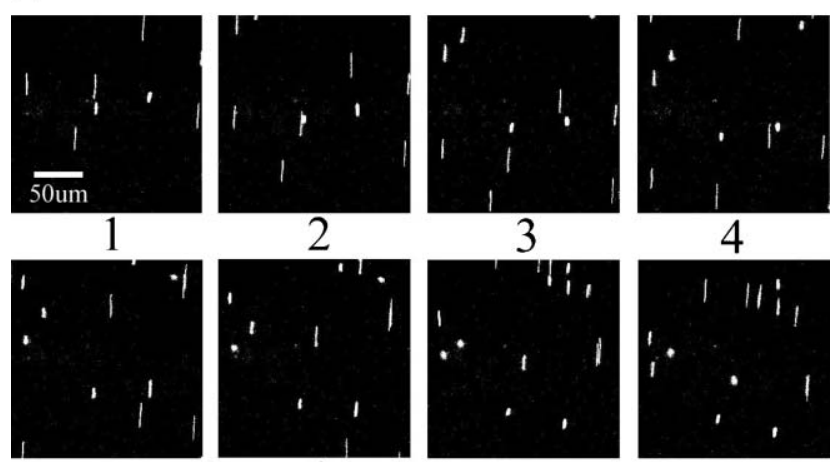
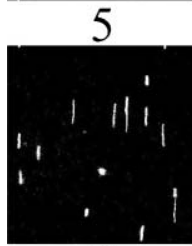

9

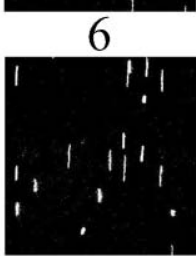

10

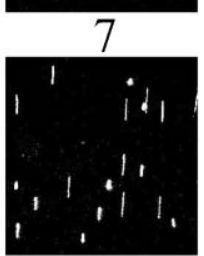

11

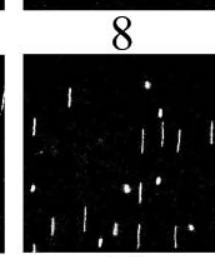

12
Fig. 3 Motion of fluorescence microbeads under hydrostatic pressure in a microchannel at 7.513 frames $\mathrm{s}^{-1}$ illumination with different excitation modes: $20 \times$ objective $(\mathrm{NA}=0.75$ ); sample, $3 \times$ $10^{-8}$ beads $\mathrm{mL}^{-1}$ in water; microchannel, $300 \mu \mathrm{m}$ wide and $10 \mu \mathrm{m}$ deep; $111 \mathrm{~ms}$ exposure time and $22.1 \mathrm{~ms}$ delay time. (a) Evanescent field excitation mode, imaging area is $57.6 \mu \mathrm{m} \times 86.4 \mu \mathrm{m}$; (b) wide field excitation mode, imaging area is $208 \mu \mathrm{m} \times 214.4 \mu \mathrm{m}$.

distance divided by the interval time of the two images. Fig. 3B demonstrates the motion of microbead illumination with wide field excitation. More fluorescence microbeads were illuminated in this case. At a suitable exposure time, the fluorescence tracks formed a trace of movement. The track length divided by exposure time would be the migration velocity of microbeads. This velocity determination method was called the streak method. ${ }^{14}$ It is clear that the trace lengths of different microbeads are obviously various. We followed 30 microbeads excited with wide field mode and calculated their velocities in every frame. The typical velocities of microbeads $v s$. normalized residency time in the view field are presented in 
Fig. 4. Three typical varieties of velocities were recorded in the view field, including steady velocity with high values (full squares), regularly changed velocity including increased velocity (full circles) and decreased velocity (full triangles), random changed velocity (open squares). The microbeads, which flowed within the region of $\pm 8 \mu \mathrm{m}$ (errors less than $10 \%$ in this region) from the center with velocities larger than those determined in evanescent field, were used as an effective microbead for calculating the maximal velocity. The maximal velocity (calculated from the images of Fig. 3B) and the near wall velocity (calculated from the images of Fig. 3A) are shown in Fig. 5. The maximal velocity in the main flow and velocity at the bottom of the channel were $253.92 \pm 7.30 \mu \mathrm{m} \mathrm{s}^{-1}$, $38.75 \pm 6.71 \mu \mathrm{m} \mathrm{s}^{-1}$ respectively.

For a channel flow, the Reynolds number is defined as $R e=D_{\mathrm{H}}\left(V_{\mathrm{av}} / v\right)$. Here, the hydraulic diameter $D_{\mathrm{H}}=(2 \mathrm{WH} /$ $(W+H))$ is the characteristic length; $W$ is the channel width, $300 \mu \mathrm{m} ; H$ is the channel depth, $10 \mu \mathrm{m} ; V_{\text {av }}$ is the average velocity, $v$ is the kinetic viscosity of water, $10^{-6} \mathrm{~m}^{2} \mathrm{~s}^{-1}$. In our experiments, $R e<0.01$, the order of magnitude for the fully developed flow length $(L e)$ scale can be estimated $L e=(0.625+$ $0.022 R e) D_{\mathrm{H}} \approx 6.25 \mu \mathrm{m} .{ }^{34}$ Hence, the laminar flow was fully developed at the imaging point $(\approx 2 \mathrm{~cm}$ away from the entry reservoir). Considering $W / H \sim 30$, the velocity profile of Poiseuille flows in two dimensions is presented as ${ }^{35}$ (Fig. 1C):

$$
V=V_{\max }\left[1-\left(\frac{2 y}{H}\right)^{2}\right]=1.5 V_{\mathrm{av}}\left[1-\left(\frac{2 y}{H}\right)^{2}\right]
$$

The microbeads could not completely be immersed into the evanescent field because the diameter of the microbead is larger than the evanescent field depth. Only the centroids of microbeads located in the range $87.5 \mathrm{~nm}$ to $187.5 \mathrm{~nm}$ from the bottom surface of the channel, could be observed. Because of the large sizes of the microbeads, different parts of one microbead seen in the evanescent field were located in various flow-velocity regions in the channel. To simplify the calculation, we only considered the translation movement of a microbead and neglected the trundle in this paper. Since the particles experience the velocity field in the range from 0 to

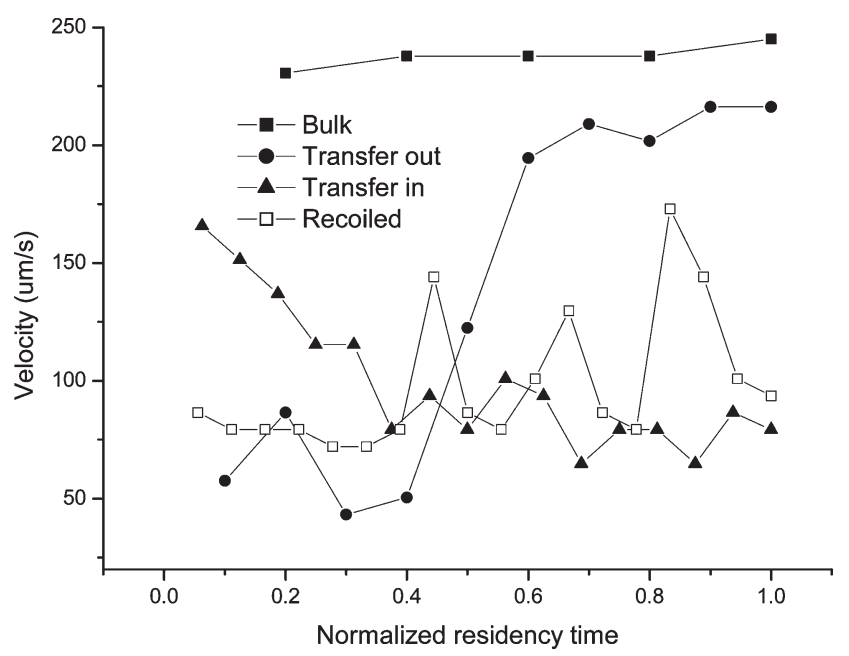

Fig. 4 Typical microbead velocities calculated from the images of Fig. 3B varied with normalized residency time in wide field mode.

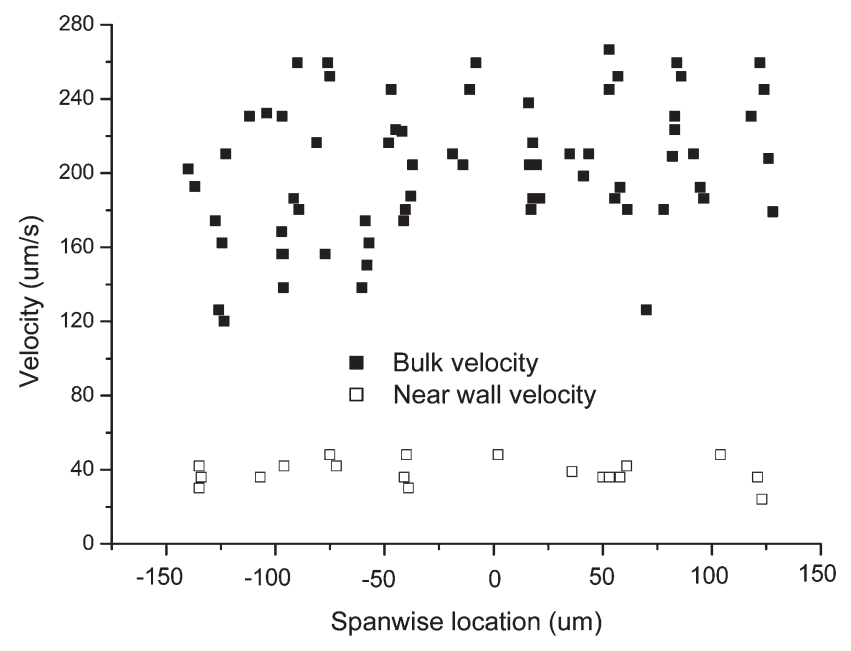

Fig. 5 Velocities of microbeads measured with wide field and evanescent field. $Z$-axis is the microbead location in the spanwise direction. (For details see text.)

$275 \mathrm{~nm}$, the particle motion measured reflects an average velocity in that range. Based on formula (2), the velocity away from walls 0 to $275 \mathrm{~nm}$ is calculated in the range from $0 \mu \mathrm{m} \mathrm{s}^{-1}$ to $27.83 \pm 0.78 \mu \mathrm{m} \mathrm{s}^{-1}$, which is comparable to the experimental results $38.75 \pm 6.71 \mu \mathrm{m} \mathrm{s}^{-1}$ in Fig. 5. The error can be further eliminated by optimizing some experimental conditions such as calibrating the laser incidence angle and increasing the quantity of captured particles.

Brownian diffusion is an important factor affecting the measurement uncertainty when sub-micron particles are used to probe flow fields. ${ }^{36,37}$ The mean square distance of diffusion is $r^{2}=2 D \Delta t$, where $\Delta t$ is the time interval and $D$ is the diffusion coefficient. The diffusion coefficient $D$ is given as $D=k T / 3 \pi \eta d_{\mathrm{p}}$ (Einstein 1905). Here, $d_{\mathrm{p}}$ is the particle diameter, $k$ is Boltzman's constant, $T$ is the absolute temperature of the fluid, and $\eta$ is the dynamic viscosity of the fluid. Therefore, the velocity due to Brownian motion over interval time can be estimated by

$$
v=\frac{r}{\Delta t}=\sqrt{\frac{2 D}{\Delta t}} .
$$

The diffusion coefficient of $175 \mathrm{~nm}$ particles in unconfined flow is $2.5 \times 10^{-8} \mathrm{~cm}^{2} \mathrm{~s}^{-1}$. During the $133.1 \mathrm{~ms}$ interval time, the velocity due to Brownian diffusion in plane for particles is $6.13 \mu \mathrm{m} \mathrm{s}^{-1}$. So that the velocity error of $175 \mathrm{~nm}$ particles in the center and near wall due to Brownian diffusion are 3.6\% and $15.8 \%$ (if the impact of hindered Brownian diffusion and the decreased diffusive uncertainty, $1 / \sqrt{N}$, where $N$ is the total number of particles on average, were considered, the error would be less than $5 \%^{37,38}$ ), respectively. Out-plane Brownian diffusion mainly causes an error of particle mismatch. The impact of hindered Brownian diffusion on the accuracy of particle-image velocimetry using evanescent-wave illumination was studied by Sadr, ${ }^{38}$ and they concluded that "the errors due to Brownian diffusion-induced particle mismatch are negligible compared with various experimental errors such uncertainties in estimating magnification and the discretization of the image by the CCD array". 


\section{Collisions of microbeads with channel walls}

The microbead marked with the double arrowhead in Fig. 3A initially appeared in the fifth image, and then vanished in the sixth frame. It reappeared from the seventh image to the tenth image with decreasing fluorescence intensity and pixel area, and became invisible again in the eleventh and twelfth images. Then it became visible in the thirteenth image. Because the penetration distance in the evanescence wave excitation mode is no more than $100 \mathrm{~nm}$, it is acceptable to assume that microbeads (175 $\mathrm{nm}$ diameter) collide with channel walls in an evanescent field. The disappearance of microbeads from the view field suggested that the microbead moves out of the evanescent field. It is expected that the microbeads that move out of the evanescent field will have a higher velocity than those in the evanescent field. The collided microbeads with walls, therefore, should show the obvious alterative trend of velocities in consecutive images in the wide field excitation mode. As matter of fact, parts of the trace lengths measured from microbeads in frames of Fig. 3B were not homogeneous, and this inhomogeneous velocity profile may partially be contributed by the interaction of microbeads with the channel walls. By following a single microbead, as demonstrated in Fig. 4, it clearly shows a changeable velocity during its flowing course except the solid square points. This phenomenon suggests that the microbead is colliding with channel walls during its presence in the view field. Moreover, nadiral velocities among the variational velocities of single microbeads are comparable with the velocities measured with evanescent wave excitation mode. The consideration of collision of microbeads with walls is self-consistent in both evanescent wave and wide field experiments. The data demonstrate that the velocity increases dramatically when a microbead transfers out of the near wall field and the opposite phenomenon happens when a microbead transfers in the near-wall field. We believe that those beads, which have inhomogeneous velocity, collided with channel walls (top surface or bottom surface). Our data indicate that the collisions decrease the bead velocity, but do not stop the flowing of microbeads, resulting in a short trace length, and that the collision process can be recorded by following the change of trace length of a single microbead.

\section{Confirmation of single $\lambda$ DNA molecules in an imaging frame and calculation of migration velocity of DNA molecules}

Fig. 6 shows the images of YOYO-I labeled $\lambda$ DNA molecules at various concentrations. The excitation power used was about $23 \mathrm{~mW}$ and the image in each frame was 421 pixels $\times$ 283 pixels. The concentrations of sample in Fig. 6-1, 2, 3, 4 were 109 pM, 1.09 pM, 0.109 pM, 0.0109 pM, respectively. The $\lambda$ DNA concentration in Fig. 6-1 was too high that a bright band was formed instead of individual spots. Segregated spots were observed in Fig. 6-2, 3, 4 when lower $\lambda$ DNA concentrations were used. The detection volume in Fig. 6 was about $0.842 \mathrm{~nL}$, which was calculated based on channel dimension and laser illumination area. The amounts of DNA molecules were calculated by multiplying the sample concentration with detection volume and Avogadro's number. The numbers of $\lambda$ DNA molecules which should appear in the detection volume corresponding to Fig. 6-1, 2, 3, 4 were
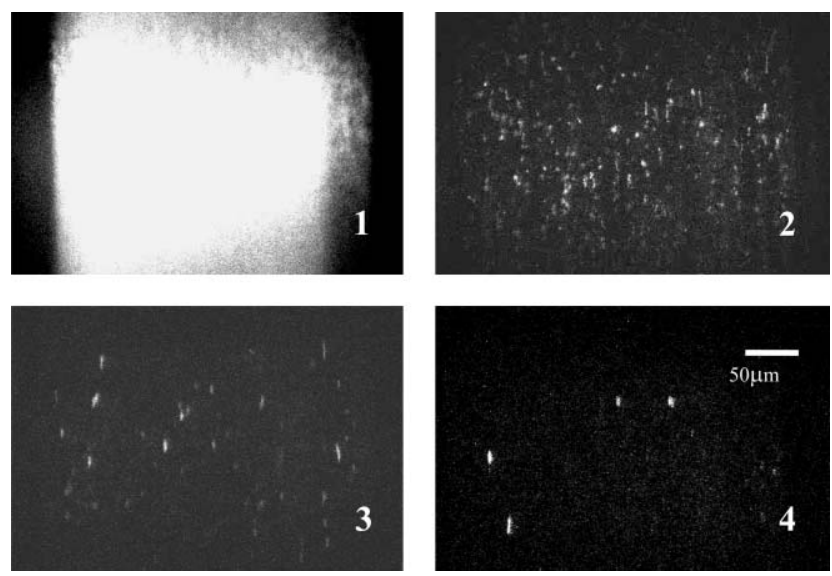

Fig. 6 Fluorescence images of YOYO-I labeled $\lambda$ DNA molecules at various concentrations. Laser power was about $23 \mathrm{~mW}$. The concentrations of $\lambda$ DNA were respectively (1) $109 \mathrm{pM}$; (2) $1.09 \mathrm{pM}$; (3) $0.109 \mathrm{pM}$; (4) $0.0109 \mathrm{pM}$. Imaging area was 421 pixels $\times 283$ pixels. Exposure time was $49 \mathrm{~ms}$. Other experimental conditions have been stated in the experimental section.

45,000, 450, 45 and 4.5, respectively. By using Fig. 6-3 as a model, five random frames were chose and the numbers of spots in each frame were counted. The average numbers of $\lambda$ DNA molecules were $39.0 \pm 3.08$ per frame. In this experiment, only one $488 \mathrm{~nm}$ notch filter was used to selectively eliminate background from scattered laser line and at $514 \mathrm{~nm}$ its transmission is about $83 \%$. The detected numbers of molecules in the detection volume should be $45 \times 83 \%=$ 37 theoretical. Therefore, the real counted spots in frame were comparable to the number of calculated molecules. These facts implied that one spot in each frame correspond to one $\lambda$ DNA molecule in our experiments.

Regarding the velocity calculation, Shortreed et al. ${ }^{14}$ have presented multiframes, streak and multispots methods to determine the migration velocity using single molecule imaging technology. They proved the migration velocity of $\lambda$ DNA molecules extracted from single molecules with the multiframes method agreed well with the ensemble analysis by capillary electrophoresis. In order to compare the multiframes methods with the streak method in the determination of migration velocity, we conducted the experiment and calculated the migration velocity of single DNA molecules with two methods respectively (Table 1). In the multiframes method, the frame frequency was $11.16 \mathrm{~Hz}$ and exposure time was $20 \mathrm{~ms}$. Fig. 7A shows the typical image of streak method at the $512 \mathrm{~ms}$ exposure time. The white numbers above the traces were the trace lengths in pixels, namely the migration distance. Fig. 7B demonstrates that various exposure time for the molecules resulted in different trace lengths for the same velocity. The slope of the line represents the migration velocity. We repeated to plot this line five times, and achieved five slopes. The results of migration velocity calculated with these two methods were not significantly different at $90 \%$ confidence level with T-test. Therefore, the migration velocity results of the streak method were comparable to those of multiframes method, but the streak method was faster and did not cause miscounting of DNA molecules. 
Table 1 Migration velocities of $\lambda$ DNA molecules using multiframes method and streak method. The experimental conditions are stated in the text and Fig. 7A

\begin{tabular}{|c|c|c|c|c|c|c|}
\hline \multicolumn{4}{|c|}{ Multiframes method } & \multicolumn{3}{|c|}{ Streak method } \\
\hline & $\begin{array}{l}\text { Velocity/ } \\
\text { pixel } \mathrm{ms}^{-1}\end{array}$ & $\begin{array}{l}\text { Average/ } \\
\text { pixel ms }\end{array}$ & $\begin{array}{l}\text { Standard } \\
\text { deviation } \\
\text { (SD) }\end{array}$ & $\begin{array}{l}\text { Slope / } \\
\text { pixel ms }\end{array}$ & $\begin{array}{l}\text { Average/ } \\
\text { pixel ms }\end{array}$ & SD \\
\hline 1 & 0.14509 & 0.14987 & 0.02943 & 0.16011 & 0.14687 & 0.01059 \\
\hline 2 & 0.10045 & & & 0.13581 & & \\
\hline 3 & 0.16741 & & & 0.1528 & & \\
\hline 4 & 0.12277 & & & 0.13637 & & \\
\hline 5 & 0.17857 & & & 0.14928 & & \\
\hline 6 & 0.15625 & & & & & \\
\hline 7 & 0.17857 & & & & & \\
\hline
\end{tabular}

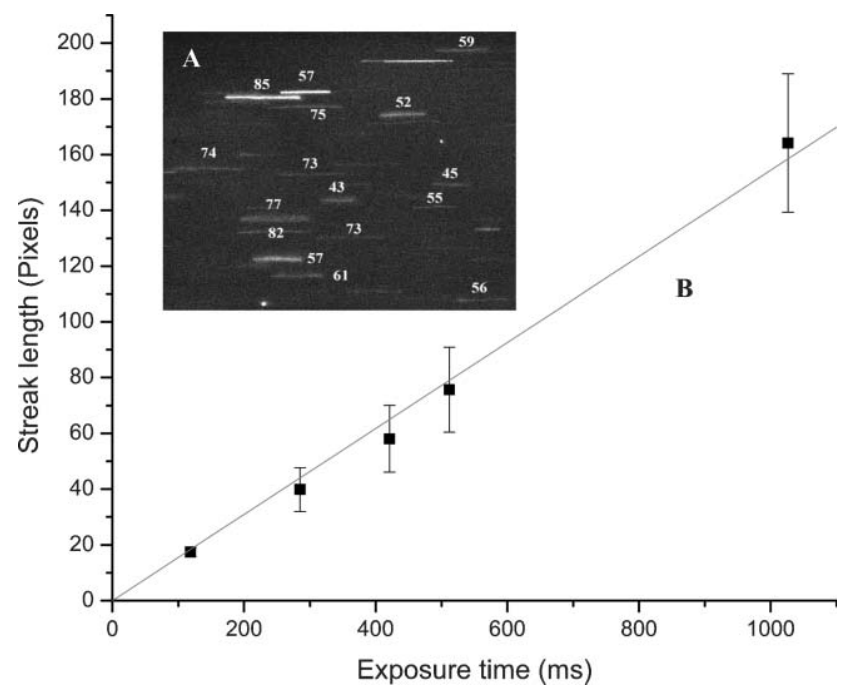

Fig. 7 (A) Typical fluorescence image of single $\lambda$ DNA molecule exposed at $512 \mathrm{~ms}$. Every bright streak indicated one $\lambda \mathrm{DNA}$ molecule. The length of every contrail labeled with the white number (unit, pixel) was the distance of migration in the image under that exposure duration. (B) The linear relationship of exposure time $v s$. the length of streaks. The slope is the velocity of $\lambda$ DNA molecules.

\section{Flow velocities observed by wide/evanescent field with $\lambda$ DNA molecules}

By using the technique we developed and the velocity calculation methods, we simultaneously measured the velocities of single $\lambda$ DNA molecules in the middle and near wall of a channel. The results are shown in Fig. 8 . A $20 \times 10.75 \mathrm{NA}$ objective was used in wide field mode and a $40 \times / 0.75$ NA objective was used in the evanescent wave mode. The data obtained by wide field demonstrated the velocity profile in a horizontal plate $(x o z)$, however, the minimum velocity was measured rather far from the wall. However with the evanescent wave mode, we can measure the velocity close to the wall. The molecular migration velocities in wide field and evanescent field were determined to be $226.41 \pm 11.77 \mu \mathrm{m} \mathrm{s}^{-1}$ and $27.09 \pm 5.67 \mu \mathrm{m} \mathrm{s}^{-1}$, respectively. By substituting the maximal bulk-flow velocity and near-wall velocity into formula (2), the distances of $\lambda$ DNA molecules from the

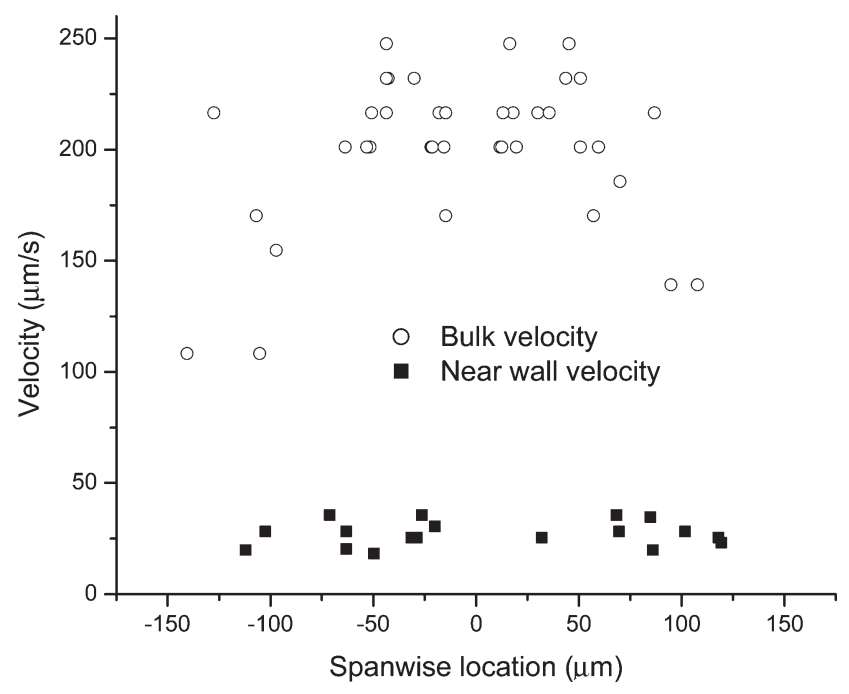

Fig. 8 Migration velocity of $\lambda$ DNA molecules measured in wide field and evanescent field.

channel surface were found to be $309.0 \pm 82.6 \mathrm{~nm}$. This result implied that the flow velocity by using evanescent field at the single molecule level, was $309.0 \pm 82.6 \mathrm{~nm}$ away from the channel wall. Its expected nearer wall velocity can be measured with smaller molecules.

\section{Conclusions}

A technique for simultaneous measuring velocities in the middle and near wall with single molecules is demonstrated. The technique allows one to selectively switch from the wide field excitation mode to the evanescent wave excitation mode. By using fluorescent microbeads with a diameter of $175 \mathrm{~nm}$ or $\lambda$ DNA molecules, the bulk-flow velocity and near-wall velocity can be determined in one single measurement. The collisions of microbeads with channel walls were directly observed and changes of velocities can be detected when a microbead transfers in and out from the near-wall field. This technique would be potentially useful for studying molecular transporting behavior in the whole flow field, and for studying interactions between molecules and microchannel surfaces, such as single molecule immunoassay.

\section{Acknowledgements}

The authors thank the financial support from NSFC of China (grant number 20275039, 2003501, 20299035, 20376080, 10272107 and "hundreds talents fund" of Chinese Academy Sciences).

Hongwei Gai, ${ }^{a}$ Ying Li, ${ }^{a}$ Zhanhua Silber-Li, ${ }^{b}$ Yinfa $\mathrm{Ma}^{* a c}$ and Bingcheng Lin*a

${ }^{a}$ Dalian Institute of Chemical Physics, Chinese Academy of Sciences, 457 Zhongshan Road, Dalian, 116023, P. R. China.

E-mail: bclin@dicp.ac.cn; Fax: +86-411-8437-9065

${ }^{b}$ State Key Laboratory for Nonlinear Mechanics, Institute of Mechanics, Chinese Academy of Sciences, No. 15 Bei Si Huan Xi Lu, Beijing 100080, P. R. China

${ }^{c}$ University of Missouri-Rolla, Rolla, MO, 65409, USA 


\section{References}

1 S. M. Nie and R. N. Zare, Annu. Rev. Biophys. Biomol. Struct., 1997, 26, 567-596.

2 S. Weiss, Science, 1999, 283, 1676-1683.

3 P. M. Goodwin, W. P. Ambrose and R. A. Keller, Acc. Chem. Res., 1996, 29, 607-613.

4 W. P. Ambrose, P. M. Goodwin, J. H. Jett, A. Van Orden, J. H. Werner and R. A. Keller, Chem. Rev., 1999, 99, 2929-2956.

5 X. S. Xie, Acc. Chem. Res., 1996, 29, 598-606.

6 X. S. Xie and J. K. Trautman, Annu. Rev. Phys. Chem., 1998, 49, $441-480$.

7 R. A. Keller, W. P. Ambrose, P. M. Goodwin, J. H. Jett, J. C. Martin and M. Wu, Appl. Spectrosc., 1996, 50, A12-A32.

8 C. Zander, Fresenius J. Anal. Chem., 2000, 366, 745-751.

9 X. H. Xu and E. S. Yeung, Science, 1997, 275, 1106-1109.

10 X. H. N. Xu and E. S. Yeung, Science, 1998, 281, 1650-1653.

11 S. H. Kang and E. S. Yeung, Anal .Chem., 2002, 74, 6234-6239.

12 J. J. Zheng and E. S. Yeung, Anal. Chem., 2002, 74, 4536-4547.

13 J. J. Zheng and E. S. Yeung, Anal. Chem., 2003, 75, 3675-3680.

14 M. R. Shortreed, H. L. Li, W. H. Huang and E. S. Yeung, Anal. Chem., 2000, 72, 2879-2885.

15 Y. F. Ma, M. R. Shortreed and E. S. Yeung, Anal. Chem., 2000, 72, 4640-4645.

16 Y. F. Ma, M. R. Shortreed, H. L. Li, W. H. Huang and E. S. Yeung, Electrophoresis, 2001, 22, 421-426.

17 A. Castro, F. R. Fairfield and E. B. Shera, Anal. Chem., 1993, 65, 849-852.

18 J. T. Petty, M. E. Johnson, P. M. Goodwin, J. C. Martin, J. H. Jett and R. A. Keller, Anal. Chem., 1995, 67, 1755-1761.

19 H. P. Chou, C. Spence, A. Scherer and S. Quake, Proc. Natl. Acad. Sci. USA, 1999, 96, 11-13.

20 A. Van Orden, R. A. Keller and W. P. Ambrose, Anal. Chem., 2000, 72, 37-41.
21 M. Sauer, B. Angerer, K. T. Han and C. Zander, Phys. Chem. Chem. Phys., 1999, 1, 2471-2477.

22 M. Ishikawa, Tanpakushitsu Kakusan Koso, 1999, 44, 2019-2023.

23 A. Femino, F. S. Fay, K. Fogarty and R. H. Singer, Science, 1998, 280, 585-590.

24 M. Gösch, H. Blom, J. Holm, T. Heino and R. Rigler, Anal. Chem., 2000, 72, 3260-3265.

25 R. J. Adrian, Ann. Rev. Fluid Mech., 1991, 23, 261-304.

26 H. A. Stone, A. D. Stroock and A. Ajdari, Ann. Rev. Fluid Mech., 2004, 36, 381-411.

27 C. D. Meinhart, S. T. Wereley and J. G. Santiago, Exp. Fluids, 1999, 27, 414-419.

28 S. Devasenathipathy, J. G. Santiago and K. Takehara, Anal. Chem., 2002, 74, 3704-3713.

29 Y. C. Kwok, N. T. Jeffery and A. Manz, Anal. Chem., 2001, 73, 1748-1753

30 C. M. Zettner and M. Yoda, Exp. Fluids, 2003, 34, 115-121.

31 R. Sadr, M. Yoda, Z. Zheng and A. T. Conlisk, J. Fluid Mech., 2004, 506, 357-367.

32 D. Axelrod, T. P. Burghardt and N. L. Thompson, Ann. Rev. Biophy. Bioeng., 1984, 13, 247-268.

33 S. H. Kang, M.R. Shortreed and E. S. Yeung, Anal. Chem., 2001, 73, 1091-1099.

34 B. Atkinson, M. P. Brocklebank, C. C. H. Card and J. M. Smith, AIChE J., 1969, 15, 548-553.

35 J. Chen, Principles of Transport Process, 1st edn., Chemical Industry Press of China, Beijing.

36 J. G. Santiago, S. T. Wereley, C. D. Meinhart, D. J. Beebe and R. J. Adrian, Exp. Fluids, 1998, 25, 316-319.

37 S. Devasenathipathy, J. G. Santiago, S. T. Wereley, C. D. Meinhart and K. Takehara, Exp. Fluids, 2003, 34, 504-514.

38 R. Sadr, H. F. Li and M. Yoda, Exp. Fluids 2004, published online: DOI: $10.1007 / \mathrm{s} 00348-004-0895-y$. 